\title{
Contactless Measuring of Temperature with Differential Photo Receiver
}

\author{
Tsanko Karadzhov \\ Department of Mechanical and \\ Precision Engineering \\ Technical University of Gabrovo \\ Gabrovo, Bulgaria \\ karadjov_s@abv.bg
}

\author{
Dimcho Pulov \\ Department of Mechanical and \\ Precision Engineering \\ Technical University of Gabrovo \\ Gabrovo, Bulgaria \\ pulov@mail.bg
}

\author{
Nikolay Angelov \\ Department of Pysics, Chamistry and \\ Ecology \\ Technical University of Gabrovo \\ Gabrovo, Bulgaria \\ angelov_np@abv.bg
}

\begin{abstract}
A method for contactless temperature measurement has been created with two photo receivers with different spectral sensitivity. An algorithm for processing the signals from both receivers of a single-chip microprocessor system has been developed. An optical system for the LWIR diapason of the spectrum is proposed.
\end{abstract}

Keywords - differential photo receiver, temperature, method, IR diapason, lens design.

\section{INTRODUCTION}

Temperature is one of the basic parameters to be measured and controlled in modern manufacture. It is increasingly important to acquire contactless methods for temperature measurement based on the registration of the thermal radiation of objects.

Planck law, Wien law, Rayleigh-Jeans law and StefanBoltzmann law are known to determine the magnitude and spectral distribution of the energetic luminosity of the heated object in terms of its temperature. These laws are used to solve the forward problem. Pyrometry is one area of science which solves the inverse problem - to find temperature of objects by the magnitude or by the spectral distribution of the energy flux emitted by them.

Two basic methods can be used to solve this problem. First method (radiation) - temperature is determined by the magnitude of the optical flux emitted by the object. Second method (method of spectral ratio) - temperature is determined by the spectral characteristics of the optical flux emitted by the object [1], [4].

At present radiation pyrometers are the largest group of non-contact temperature measurement devices. However, they have a few significant limitations. The main limitation is the dependence of the flux falling on the pyrometer from two unknown quantities: the actual temperature of the object $T_{o b j}$ (to be measured) and its emissivity coefficient $\breve{a}$.

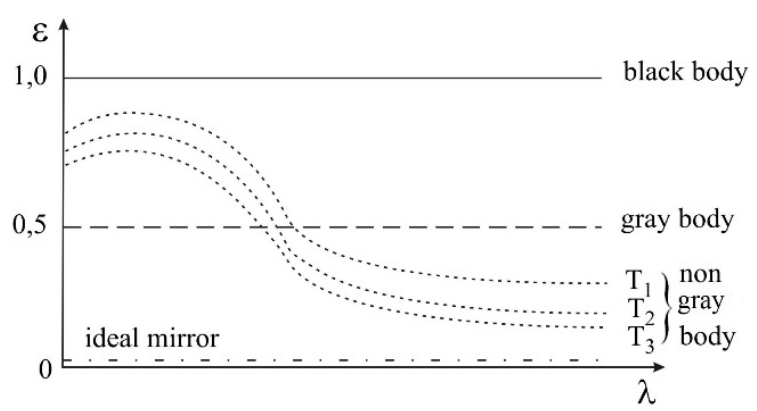

Fig. 1. Emissivity coefficient of different bodies

Emissivity coefficient of all bodies can be varied from $0 \%$ for ideal mirrors and up to $100 \%$ for black bodies (fig. 1).

Most of the measured objects are "non-gray" bodies. Therefore, their emissivity coefficient depends on the wavelength $\ddot{e}$. In addition, the emissivity coefficient depends on the temperature of the object $T_{o b j}$. Therefore $\varepsilon=f(\lambda, T)$. This implies uncertainty in the measurement results and difference $\Delta T$ between the actual temperature of the object $T_{o b j}$ and the measured one $T_{\text {meas }}\left(T_{\text {meas }}=T_{o b j}-\Delta T\right)$. The dependence $T_{o b j}=\frac{T_{\text {meas }}}{\sqrt[4]{\varepsilon(T)}}$, or $\frac{\Delta T}{T_{o b j}}=1-\sqrt[4]{\varepsilon(T)}$

is valid for radiation pyrometers where $\varepsilon(T)$ is the integral emissivity coefficient in the operational spectral diapason of device $\Delta \lambda$.

$$
\begin{aligned}
& \text { If } \varepsilon(T)=100 \% \text {, then } \frac{\Delta T}{T}=0 ; \\
& \varepsilon(T)=85 \%, \text { then } \frac{\Delta T}{T}=4 \% ; \\
& \varepsilon(T)=40 \% \text {, then } \frac{\Delta T}{T}=20 \% ;
\end{aligned}
$$

As can be seen, the error due to the unknown emissivity coefficient can be great. Therefore the value

Print ISSN 1691-5402

Online ISSN 2256-070X

http://dx.doi.org/10.17770/etr2019vol3.4132

(C) 2019 Tsanko Karadzhov, Dimcho Pulov, Nikolay Angelov. Published by Rezekne Academy of Technologies.

This is an open access article under the Creative Commons Attribution 4.0 International License. 
$\varepsilon(T)$ of the object being measured is introduced to radiation pyrometers.

The actual value of the coefficient $\varepsilon(T)$ is determined by the equation

$$
\varepsilon(T)=\frac{\int_{\Delta \lambda} \varepsilon(\lambda, T) s(\lambda) R_{\text {b }}(\lambda, T) d \lambda}{\int_{\Delta \lambda} s(\lambda) R_{b}(\lambda, T) d \lambda},
$$

where $\varepsilon(\lambda, T)$ is a spectral emissivity coefficient of the object, $s(\lambda)$ - spectral sensitivity of the photo receiver used in the pyrometer, $R_{b b}(\lambda, T)$ - spectral density of the energetic luminosity of an black body (BB).

There is ambiguity in determining the coefficient $\varepsilon(T)$ due to its complex dependence on $\lambda$ and $T$ - Therefore it is practically impossible to enter this coefficient correctly when using radiation pyrometers and the error in temperature measurement can reach tens of percent [3].

Pyrometers of spectral ratio (PSR), which are the subject of the present paper, have no such limitations.

The purpose of the present paper is to introduce a twospectral pyrometer (dual spectrum pyrometer or pyrometer of spectral ratio) in order to increase the accuracy of contactless temperature measurement as compared to the commonly used radiation pyrometers.

The goal set can be reached by accomplishing the following tasks:

- Choice and development of a functional block chart of the pyrometer;

- Development of a basic electrical circuit diagram of the pyrometer;

- Development of a device control algorithm.

\section{EXPOSITION}

\section{The essence of the two-spectral method}

The method consists in measuring the ratio of the signals from the controlled object for two narrow spectral ranges and comparing it with the ratio of the signals for the same spectral range (diapason), but obtained for an black body (fig. 2).

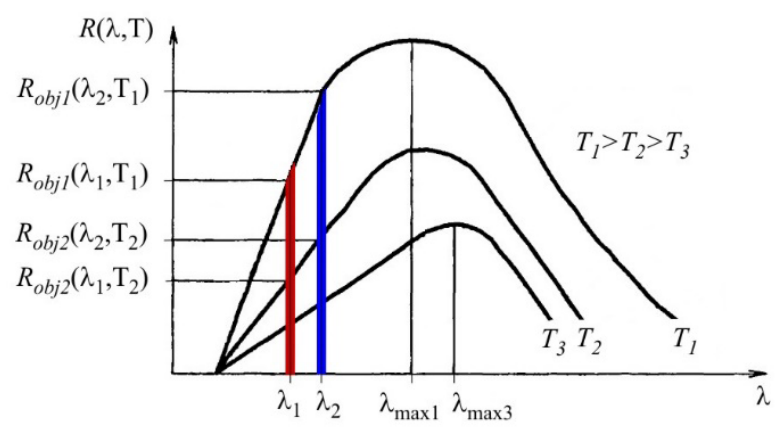

Fig. 2. The essence of the spectral method

The temperature measured $T_{\text {meas }}$ is numerically equal to the temperature of the BB (black body) where the ratio of the spectral densities $R_{b b}(\lambda, T)$ for two narrow spectral ranges is equal to the same ratio, but for the controlled object at its actual temperature $T_{o b j}$.

Therefore, the ratio between the electrical signals at the output of the two photo receivers can be expressed as follows:

$$
\begin{aligned}
& \frac{u_{1}}{u_{2}}=\frac{R_{b b}\left(\lambda_{1}, T_{\text {meas }}\right)}{R_{b b}\left(\lambda_{2}, T_{\text {meas }}\right)}=\frac{R_{o b j}\left(\lambda_{1}, T_{o b j}\right)}{R_{o b j}\left(\lambda_{2}, T_{o b j}\right)}= \\
& =\frac{R_{b b}\left(\lambda_{1}, T_{o b j}\right) \varepsilon_{\lambda_{1}}}{R_{b b}\left(\lambda_{2}, T_{o b j}\right) \varepsilon_{\lambda_{2}}} .
\end{aligned}
$$

In (1) the spectral densities $R$ will be expressed by the law of Wein, which is valid for the short wavelength range of the spectrum (under the condition that $\lambda \cdot T<C_{2}$ ).

$$
R(\lambda, T)=C_{1} \lambda^{-5} \exp ^{-\frac{C_{2}}{\lambda T}}
$$

From (1) and (2) an equation can be obtained for the relationship between the actual and the measured object temperature

$$
\frac{1}{T_{o b j}}-\frac{1}{T_{\text {meas }}}=\frac{\lambda_{1} \lambda_{2}}{C_{2}\left(\lambda_{2}-\lambda_{2}\right)} \cdot \ln \left(\varepsilon_{1} / \varepsilon_{2}\right) .
$$

From (3) follows that:

- For gray bodies $T_{o b j}=T_{\text {meas }}$. Certain bodies that are in two close spectral diapasons can be accepted as gray;

- For non-gray bodies the correct determination of $T_{o b j}$ depends not on the absolute value of the refractive index, but on the ratio $\varepsilon_{1} / \varepsilon_{2}$. This ratio is more stable when changing the external conditions by changing the absolute value of the integral emissivity coefficient $\varepsilon(T)$ used in radiation pyrometers.

\section{Choice and development of a function circuit di- agram of a two-spectral pyrometer.}

The function circuit of the two-spectral pyrometer can be created in two variants depending on the means of signal generation in the two spectral diapasons. In the first case a beam splitting device and two separate photo receivers are used. In the second case one tandem structured photo receiver ("sandwich" type) is used. In the latter case no beam splitting device is used. There is a different dependence of the current in dark environment from the temperature for the two spectral diapasons. This will increase the measurement error. For this reason, the circuit with two separate photo receivers and a beam splitting device is used in the developed pyrometer.

Fig. 3 shows the function circuit of the developed two-spectral pyrometer. The heated object emits a flux in a hemisphere. Part of the flux falls on the pyrometer's lens and focuses on the beam splitting device. 


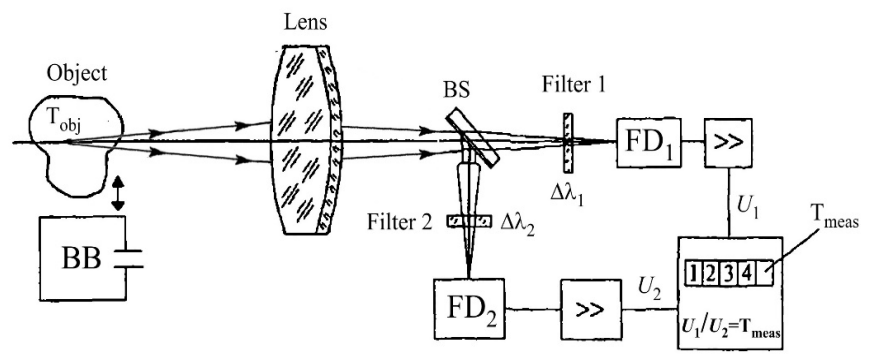

Fig. 3.

The lens is an achromatic doublet AC254-100B-ML from the Thorlabs catalog. The beamsplitter is an interference filter which partially passes and partially reflects the radiation. About of beamsplitter was selected a translucent sheet BSW11 from the Thorlabs catalog. It is made of quartz glass (Fused Silica) and divides the optical flow in a 50:50 ratio. The device divides the optical signal between the two photo receivers. Each photo receiver has a light filter. Two identical silicone photodiodes are selected as photo receivers Hamamatsu S2386. As filters from the catalog of Thorlabs are selected accordingly: filter 1 - FB900-10 (Center Wavelength $C W=900 n m$; Full Width Half Max PWHM=10nm and filter 2 - FB1000-10 $(C W=1000 \mathrm{~nm} ; P W H M=10 \mathrm{~nm})$. The photodiodes produce electrical signals proportional to the optical flux. These signals are amplified in an electronic processing unit, and then their ratio is determined which is subsequently converted to a value of the measured temperature of the controlled object $T_{\text {meas }}$.

\section{Development of basic electrical circuit of the two-spectral pyrometer.}

Fig. 4 shows the basic electrical circuit of the device. Silicon photodiodes are used as photo receivers.

The photoelectric current $I_{p h}$ through the photodiodes is transformed into a voltage by means of feedback amplifiers LM258:

$$
U_{0}=I_{p h} \cdot R_{f}
$$

where $U_{0}$ is the voltage at the output of the amplifier, $R_{f}$ - feedback resistance.

The feedback of the operational amplifier is selected so as to use the entire measurement range of the analog-todigital converter of the controller.

The 8-bit PIC18F452 microcontroller which has a 10bit analog-to-digital converter is used to control the device operation. The controller has a sufficient number of inputoutput ports and a sufficient amount of program memory where the control program can be located.

The threshold voltages that determine the measurement range of the converter are respectively $\mathrm{V}_{\mathrm{REF}}=0 \mathrm{~V}$ and $\mathrm{V}_{\mathrm{REF}}+$ $=5 \mathrm{~V}$. The input analog signal to ADC is expressed as $2^{10}$ or 1024 levels. The increase in voltage is determined by the formula:

$$
\Delta U=\frac{V_{R E F+}}{2^{10}}=\frac{5}{1024}=4,883 \mathrm{mV}
$$

The device functional circuit consists of two measuring channels. The voltage from the outputs of the currentto-voltage converters is fed to two analog inputs of the controller RA0/AN0 and RA1/AN1. The inputs switch. At any moment of time voltage is measured at one input only.

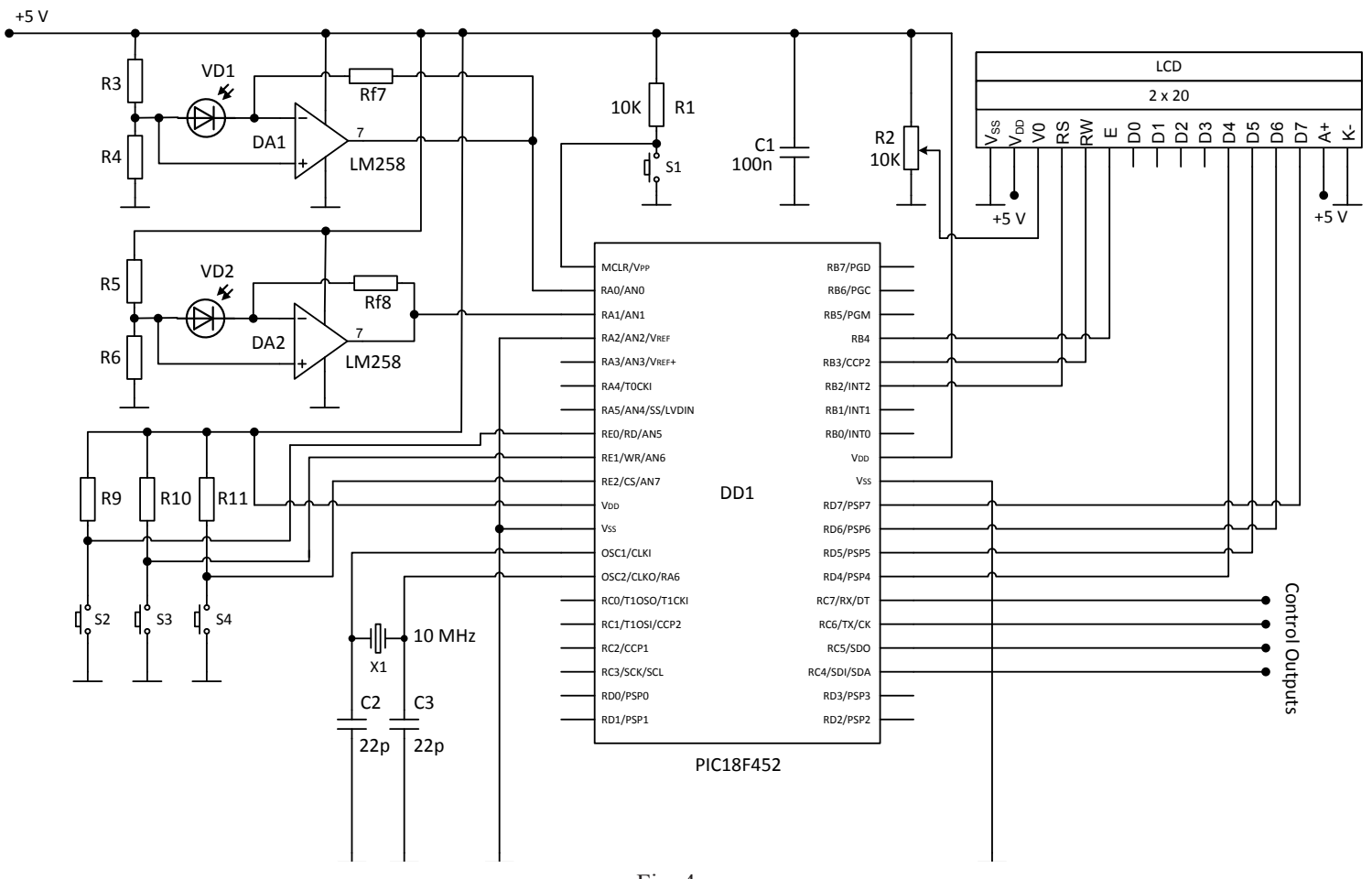

Fig. 4. 


\section{Development of a block chart of the program al- gorithm.}

The block chart of the control program algorithm is shown in fig. 5. [7]. First, the controller is initialized, constants and variables are defined and corresponding cells from the data memory are reserved for them. The next stage of the block chart is related to determining the $\mathrm{I} / \mathrm{O}$ ports to be used. Digital inputs, analog inputs and digital outputs are determined. PIC18F452 has 4 internal module timers. Only Timer 0 is used. It sets time lags in the program which are used to synchronize the operation of the microcontroller and the alphanumeric LCD. Next, an initialization of the display is performed. The master program is a continuous loop, which stops only when the voltage measurement from the ADC (analog-to-digital converter) has been completed.

The processing of the result of each measurement takes place in a subroutine, which starts when an ADC break occurs.

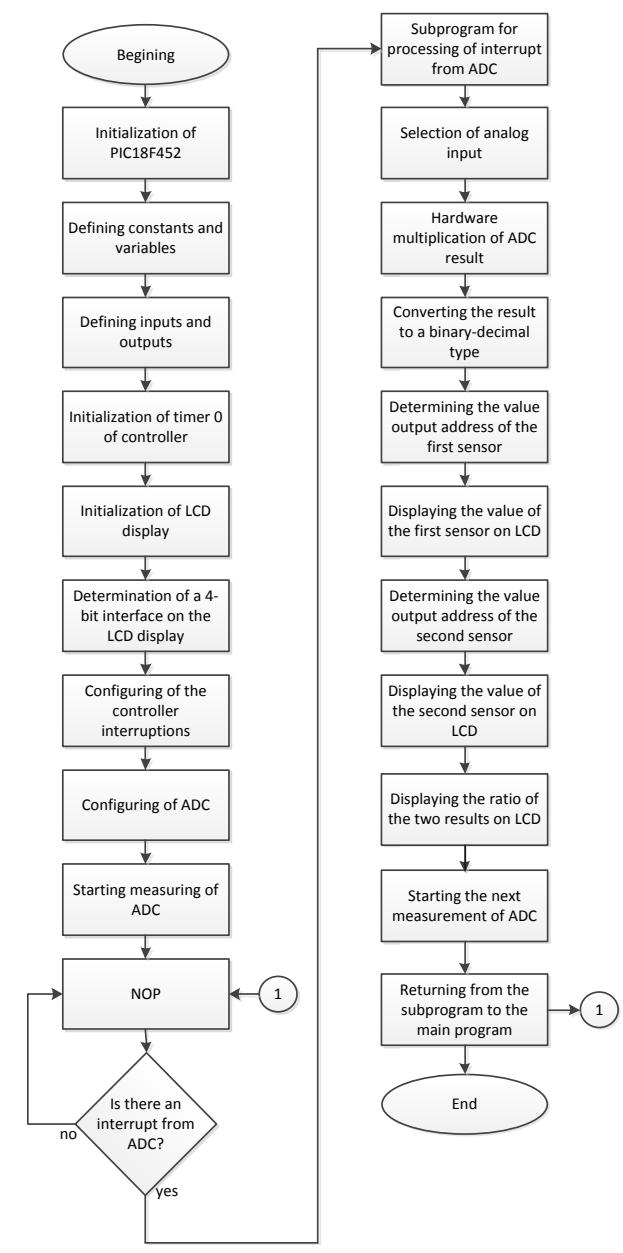

Fig. 5. Control program algorithm

\section{Subroutine for processing ADC break}

At the beginning of the subroutine an analog input for the next measurement is selected. The result of the previous measurement is then processed, which includes hardware multiplication of the measurement value by a preset constant. The ratio $U_{1} / U_{2}$ between the voltages measured in the two channels is determined. The result is converted from hexadecimal to binary-decimal code.

The second stage of the subroutine is related to out- putting the measurement results on an LCD display. First, the output addresses are defined, it means - to determine in which position to display each digit from the result, including the decimal points. The values of the calculations are then displayed. Finally, a subsequent measurement is started in the subroutine and then returning to the master program takes place.

\section{Connecting to the microcontroller and controlling} the LCD display

The display used is a two-line, alphanumeric, liquid crystal display, with 20 characters per line. It is a smart display with its own control scheme-LCD driver hd44780. The display has a total of 16 ports to enable power supply and connection to an external microcontroller. For controlling the LCD module a control bus is used comprising RS, $\mathrm{R} / \mathrm{W}$, E ports which are correspondingly connected to microcontroller ports RB2, RB3, RB4 initialized as digital outputs. The data bus of this type of display consists of 8 ports. The microcontroller communicates with the LCD module by means of the data bus. In this case a mode is selected for this communication to take place using 4-bit interface. The DB4, DB5, DB6, DB7 display ports are initialized as inputs and correspondingly connected to the microcontroller's RD4, RD5, RD6, RD7 ports which are initialized as outputs. At the beginning of the program, initialization of the display takes place, which selects the following:

- 4-bit interface;

- two-line mode;

- character format 5x8 dots;

- incrementing the address counter by one unit when in $\mathrm{read} / \mathrm{record}$ mode

\section{CONCLusion}

A two-spectral pyrometer with two identical silicon photodiodes and a beam splitter has been developed. The use of this circuit reduces the influence of external conditions on the measurement error. The device is intended for high temperature measurements ranging from $700{ }^{\circ} \mathrm{C}$ to $1500{ }^{\circ} \mathrm{C}$. The spectral diapasons of the two channels are separated by narrowband filters, thus increasing the accuracy of the measurement.

\section{REFERENCES}

[1] Lisiyenko V.G., Shleymovich YE.M., Ladygichev M.G., Sannikov S.P., Shchelokov Ya.M. Temperatura: teoriya, praktika, eksperiment. Spravochnoye izdaniye: V 3-kh tomakh. T.1, kniga 2 - M.: Teplotekhnik, 2009 - 340 s.

[2] Frunze A.V. Vliyaniye metodicheskikh pogreshnostey pirometra na vybor pribora, Fotonika - 2012. - № 3 - S.46-51; № 3 - S.5660.

[3] Frunze A.V. Pirometry spektral'nogo otnosheniya: preimushchestva, nedostatki i puti ikh ustraneniya, Fotonika 2009. -№ 4 - S.32-37.

[4] Belen'kiy A.M., Dubinskiy M.Yu., Ladygichev M.G., Lisiyenko V.G., Shchelokov Ya.M. Izmereniye temperatury: teoriya, praktika, eksperiment. Spravochnoye izdaniye: V 3-kh tomakh. T.2 - M.: Teplotekhnik, $2007-736$ s.

[5] A. Sala, Radiant properties of materials, Elsevier Publishing, Oxford, 1986

[6] Lyubomir Lazov, Nikolay Angelov, Numerical experiments for determining of laser inducion temperature fields, Turkish Journal of Physics, 37, 212-218, Turkey, 2013

[7] Karadzhov, Ts. V., I. S. Balabanova, M. S. Slavov, Multi-channel electronic device for temperature monitoring. Elektrotechnica \& Elektronica, Vol. 48. No 1-2/2013, ISSN 0861-4717.

[8] Chrzanowski, K., Non-Contact Thermometry. Measurement Errors. SPIE Polish Chapter, Warsaw, 2001 